\title{
An Improved Ranking Strategy for Fuzzy Multiple Attribute Group Decision Making
}

\author{
Zhanhong Hu, Zichun Chen, Zheng Pei, Xinzi Ma, Wei Liu \\ School of Mathematic \& Computer Engineering, Xihua University, \\ Chengdu, Sichuan, 610039, China \\ E-mail:pqyz@263.net \\ Received 23 March 2012 \\ Accepted 16 October 2012
}

\begin{abstract}
In this paper, we develop an improved ranking strategy for fuzzy multiple attribute group decision making. First, we introduce a method for multiple attribute group decision making and show that method can not choose the best alternative, when $S\left(\widetilde{P}_{i} \ominus \widetilde{P}_{j}\right)=0$ and $\widetilde{P}_{i} \neq \widetilde{P}_{j}$. We define then a k-order deviation of the fuzzy sets and propose an improved ranking strategy, we also propose an improved method for fuzzy multiple attribute group decision making. Finally, the results of a numerical example demonstrate the effectiveness of this new method.
\end{abstract}

Keywords: Ranking strategy, Fuzzy group decision making, FIOWA operators, The best alternative

\section{Introduction}

Multiple attribute decision making (MADM) problems are wide spread in real-life decision making situation ${ }^{1}$. A multiple attribute decision making problem is to find a desirable solution from a finite number of feasible alternatives assessed on multiple attributes, both quantitative and qualitative. In order to choose a desirable solution, decision maker often provide his/her preference information which takes the form of numerical values, such as exact values, interval number values and fuzzy numbers. However, under many conditions, numerical values are inadequate or insufficient to model real-life decision problems. Indeed, human judgments including preference information may be stated in linguistic terms. Thus, multiple attribute decision making problems under linguistic environment is an interesting research topic having received more and more atten- tion from researchers during the last several years. In the process of multiple attribute decision making, the linguistic decision information needs to be aggregated by means of some proper approaches so as to rank the given decision alternatives and then to select the most desirable one. Wang et al. ${ }^{14}$ presented the Up and Lo operators which satisfy the partial ordering relations of fuzzy numbers for generalizing the TOPSIS for handling fuzzy multiple-criteria group decision making problems. Liao et al. ${ }^{6}$ presented a model for selecting an ERP system based on linguistic information processing. Chou et al. ${ }^{5}$ attempted to fill the gap in the current literature by establishing a fuzzy multiple-criteria decision making (FMCDM) model for the risk evaluation of investment location. Pei et al. ${ }^{11,12}$ presented linguistic weighted aggregation operator to handle fuzzy risk analysis. Zhang et al. ${ }^{19}$ presented a method to handle fuzzy group decision making based on house of 
quality for multi-format and multi-granularity linguistic judgments in quality function deployment. Rodríguez et al ${ }^{13}$ presented a multicriteria linguistic decision making model in which experts provide their assessments by eliciting linguistic expressions. The decision model manages such linguistic expressions by means of its representation using hesitant fuzzy linguistic term sets. Martínez et al. ${ }^{8}$ made an overview on the 2-tuple linguistic model for computing with words in decision making: extensions, applications and challenges.

In recent years, some researchers handle fuzzy multiple attribute group decision making problems using OWA operators. Yager ${ }^{17}$ presented the ordered weighted averaging (OWA) aggregation operators to handle decision making problems. Yager and Filev ${ }^{18}$ extended the OWA operators to present the induced ordered weighted averaging (IOWA) operators. Chiclana et al. ${ }^{3}$ presented induced ordered weighted averaging operators for handling group decision making problems based on fuzzy preference relations. Palomares et al. ${ }^{10}$ studied the concept of groups attitude towards consensus, and presented a consensus model that integrates it in the measurement of consensus, through an extension of OWA aggregation operators, the so-called Attitude-OWA. Chen et al. ${ }^{4}$ presented a method for handling multicriteria fuzzy decision making problems using FNIOWA operators. $\mathrm{Xu}{ }^{16}$ presented induced uncertain linguistic OWA (IULOWA) operators for handling group decision making problems. Chen et al. ${ }^{2}$ present a new approach for fuzzy multiple attribute group decision making based on the proposed fuzzy induced ordered weighted averaging (FIOWA) operators. Merigo et al. ${ }^{7}$ developed a new decision making model using the Dempster-Shafer (DS) belief structure when available information is uncertain and can be assessed with interval numbers. Wei ${ }^{15}$ presented a method based on the ET-WG and ET-OWG operators for multiple attribute group decision-making, in the approach, alternative appraisal values are calculated by the aggregation of 2-tuple linguistic information. Su et al. ${ }^{9}$ developed an approach based on the induced generalized intuitionistic fuzzy ordered weighted averaging (IGIFOWA) and intuitionistic fuzzy weighted averaging
(IFWA) operators to solve multiple attribute group decision making (MAGDM) problems with intuitionistic fuzzy information. In this paper, we focus on Chen's method ${ }^{2}$. We find that this method can not choose the best alternative, when $S\left(\widetilde{P}_{i} \ominus \widetilde{P}_{j}\right)=0$ and $\widetilde{P}_{i} \neq \widetilde{P}_{j}$. In order to solve this problem, we develop an improved ranking strategy. We define the k-order deviation of the fuzzy sets, and prove two important theorems. An improved method for fuzzy multiple attribute group decision making is then proposed.

The rest of this paper is organized as follows. In Section 2, we briefly review the ranking strategy of Chen's method ${ }^{2}$ and show " $\leqslant s$ " is not an partial order relation of fuzzy sets. In Section 3, an improved ranking is proposed. In Section 4, we develop an improved method for fuzzy multiple attribute group decision making. In Section 5, we give an illustrative example to verify the developed approach and to demonstrate its feasibility and practicality. The conclusions are discussed in Section 6.

\section{Fuzzy Multiple Attribute Group Decision Making Based on FIOWA Operators}

We consider a finite and totally ordered discrete linguistic label set $S=\left\{s_{i} \mid i=-m, \cdots,-1,0,1 \cdots, m\right\}$, where $m$ is a positive integer, $s_{i}$ represents a possible value for a linguistic variable and satisfies $s_{i}>s_{j}$ if $i>j$. For example, a set of nine terms $S$ could be $S=\left\{s_{-4}=\right.$ extremely poor, $s_{-3}=$ very poor, $s_{-2}=$ poor,$s_{-1}=$ slightly poor, $s_{0}=$ fair,$s_{1}=$ slightly good, $s_{2}=$ good, $s_{3}=$ very good, $s_{4}=$ extremely good $\}$.

Definition 1. ${ }^{2}$ Let $\widetilde{P}_{1}=a_{1} / s_{B_{1}}+a_{2} / s_{B_{2}}+\cdots+$ $a_{n} / s_{B_{n}}, \widetilde{P}_{2}=b_{1} / s_{B_{1}}+b_{2} / s_{B_{2}}+\cdots+b_{n} / s_{B_{n}}$, be two fuzzy sets, where $B_{1}, B_{2}, \cdots, B_{n}$ are integers, $B_{1}<$ $B_{2}<\cdots<B_{n}, s_{B_{1}}<s_{B_{2}}<\cdots<s_{B_{n}}, a_{i}$ denotes the grade of membership of $s_{B_{i}}$ in the fuzzy set $\widetilde{P}_{1}, b_{j}$ denotes the grade of membership of $s_{B_{j}}$ in the fuzzy set $\widetilde{P}_{2}, a_{i} \in[0,1], b_{j} \in[0,1], 1 \leqslant i \leqslant n$ and $1 \leqslant j \leqslant n$.

The addition operation between the fuzzy sets $\widetilde{P}_{1}$ 
and $\widetilde{P}_{2}$ is

$$
\begin{aligned}
\widetilde{P}_{1} \oplus \widetilde{P}_{2}= & \left(a_{1} / s_{B_{1}}+a_{2} / s_{B_{2}}+\cdots+a_{n} / s_{B_{n}}\right) \\
& \oplus\left(b_{1} / s_{B_{1}}+b_{2} / s_{B_{2}}+\cdots+b_{n} / s_{B_{n}}\right) \\
= & \left(a_{1}+b_{1}\right) / s_{B_{1}}+\left(a_{2}+b_{2}\right) / s_{B_{2}} \\
& +\cdots+\left(a_{n}+b_{n}\right) / s_{B_{n}} .
\end{aligned}
$$

The multiplication operation between $\alpha$ and $\widetilde{P}_{1}$ is

$$
\begin{aligned}
\alpha \otimes \widetilde{P}_{1}= & \alpha \otimes\left(a_{1} / s_{B_{1}}+a_{2} / s_{B_{2}}+\cdots+a_{n} / s_{B_{n}}\right) \\
= & \left(\alpha \times a_{1}\right) / s_{B_{1}}+\left(\alpha \times a_{2}\right) / s_{B_{2}} \\
& +\cdots+\left(\alpha \times a_{n}\right) / s_{B_{n}} .
\end{aligned}
$$

Definition 2. ${ }^{2}$ A fuzzy induced OWA operator is defined as follows:

$$
\begin{array}{r}
F_{\text {FIOWA }}\left(\left\langle u_{1}, \widetilde{s}_{1}\right\rangle,\left\langle u_{2}, \widetilde{s}_{2}\right\rangle, \cdots,\left\langle u_{n}, \widetilde{s}_{n}\right\rangle\right) \\
=w_{1} \widetilde{S}_{b 1} \oplus w_{2} \widetilde{S}_{b 2} \oplus \cdots \oplus w_{n} \widetilde{S}_{b n},
\end{array}
$$

where $\mathrm{W}=\left(w_{1}, w_{2}, \cdots, w_{n}\right)^{T}$ is a weighting vector, such that $w_{j} \in[0,1], \sum_{j=1}^{n} w_{j}=1 . u_{i}$ in the OWA pair $\left\langle u_{i}, \widetilde{s}_{i}\right\rangle$ is called the order inducing variable, $\widetilde{s_{i}}$ is called the uncertain linguistic argument variable, $\widetilde{S}_{b j}$ is the value of the OWA pair having the jth largest order inducing $u$ value, $1 \leqslant i \leqslant n$, and $1 \leqslant j \leqslant n$.

Definition 3. ${ }^{2}$ Let $\widetilde{P}_{1}=a_{1} / s_{B_{1}}+a_{2} / s_{B_{2}}+\cdots+$ $a_{n} / s_{B_{n}}$ and $\widetilde{P}_{2}=b_{1} / s_{B_{1}}+b_{2} / s_{B_{2}}+\cdots+b_{n} / s_{B_{n}}$ be two fuzzy sets, where $B_{1}, B_{2}, \cdots, B_{n}$ are integers, $B_{1}<B_{2}<\cdots<B_{n}, s_{B_{1}}<s_{B_{2}}<\cdots<s_{B_{n}}, a_{i}$ denotes the grade of membership of $s_{B_{i}}$ in the fuzzy set $\widetilde{P}_{1}, b_{j}$ denotes the grade of membership of $s_{B_{j}}$ in the fuzzy set $\widetilde{P}_{2}, a_{i} \in[0,1], b_{j} \in[0,1], 1 \leqslant i \leqslant n$ and $1 \leqslant j \leqslant n$. Then, the score $S\left(\widetilde{P}_{1} \ominus \widetilde{P}_{2}\right)$ of the weighted difference of the membership values between the fuzzy sets $\widetilde{P}_{1}$ and $\widetilde{P}_{2}$ is defined by

$$
\begin{aligned}
S\left(\widetilde{P}_{1} \ominus \widetilde{P}_{2}\right)= & \left(a_{1} / s_{B_{1}}+\cdots+a_{n} / s_{B_{n}}\right) \\
& \ominus\left(b_{1} / s_{B_{1}}+\cdots+b_{n} / s_{B_{n}}\right) \\
= & B_{1} \times\left(a_{1}-b_{1}\right)+B_{2} \times\left(a_{2}-b_{2}\right) \\
& +\cdots+B_{n} \times\left(a_{n}-b_{n}\right) .
\end{aligned}
$$

Formally, the above definition provides an order relation between two fuzzy sets on linguistic term set.

Definition $4 .^{2}$ Let $\widetilde{P}_{1}, \widetilde{P}_{2}$ and $\widetilde{P}_{3}$ be three fuzzy sets in the universe of discourse $U$, where $U=$ $\left\{s_{B_{1}}, s_{B_{2}}, \cdots, s_{B_{n}}\right\}$. If $S\left(\widetilde{P}_{1} \ominus \widetilde{P}_{1}\right) \geqslant S\left(\widetilde{P}_{1} \ominus \widetilde{P}_{2}\right)$, then $\widetilde{P}_{1} \leqslant \widetilde{P}_{2} . \quad$ If $S\left(\widetilde{P}_{1} \ominus \widetilde{P}_{2}\right) \geqslant S\left(\widetilde{P}_{1} \ominus \widetilde{P}_{3}\right)$, then $\widetilde{P}_{2} \leqslant$ $\widetilde{P}_{3}$. If $S\left(\widetilde{P}_{1} \ominus \widetilde{P}_{1}\right) \geqslant S\left(\widetilde{P}_{1} \ominus \widetilde{P}_{2}\right)$ and $S\left(\widetilde{P}_{1} \ominus \widetilde{P}_{2}\right) \geqslant$ $S\left(\widetilde{P}_{1} \ominus \widetilde{P}_{3}\right)$, then $\widetilde{P}_{1} \leqslant \widetilde{P}_{2} \leqslant \widetilde{P}_{3}, \min _{i=1,2,3}\left(\widetilde{P}_{i}\right)=\widetilde{P}_{1}$, $\max _{i=1,2,3}\left(\widetilde{P}_{i}\right)=\widetilde{P}_{3}$.

Next, we analyze Chen's method, and find that it can not choose the best alternative, when $S\left(\widetilde{P}_{i} \ominus\right.$ $\left.\widetilde{P}_{j}\right)=0$ and $\widetilde{P}_{i} \neq \widetilde{P}_{j}$.

Theorem 1. For any fuzzy set $\widetilde{P}_{i}=c_{1} / s_{B_{1}}+$ $c_{2} / s_{B_{2}}+\cdots+c_{n} / s_{B_{n}}, \widetilde{P}_{j}=d_{1} / s_{B_{1}}+d_{2} / s_{B_{2}}+\cdots+$ $d_{n} / s_{B_{n}}$ and $\widetilde{P}_{k}=e_{1} / s_{B_{1}}+e_{2} / s_{B_{2}}+\cdots+e_{n} / s_{B_{n}}$ on $U=\left\{s_{B_{1}}, s_{B_{2}}, \cdots, s_{B_{n}}\right\}, " \leqslant s$ " satisfies reflexivity and transitivity, i.e., (1) Reflexivity: $\widetilde{P}_{i} \leqslant_{s} \widetilde{P}_{i} ;$ (2) Transitivity: If $\widetilde{P}_{i} \leqslant s \widetilde{P}_{j}, \widetilde{P}_{j} \leqslant s \widetilde{P}_{k}$, then $\widetilde{P}_{i} \leqslant s \widetilde{P}_{k}$. Proof. (1) Due to $S\left(\widetilde{P}_{i} \ominus \widetilde{P}_{i}\right) \leqslant S\left(\widetilde{P}_{i} \ominus \widetilde{P}_{i}\right)=0, \widetilde{P}_{i} \leqslant s \widetilde{P}_{i}$ is obvious.

(2) $\widetilde{P}_{i} \leqslant s \widetilde{P}_{j}$ if and only if $S\left(\widetilde{P}_{i} \ominus \widetilde{P}_{j}\right) \leqslant 0, \widetilde{P}_{j} \leqslant s \widetilde{P}_{k}$ if and only if $S\left(\widetilde{P}_{j} \ominus \widetilde{P}_{k}\right) \leqslant 0 . \quad S\left(\widetilde{P}_{i} \ominus \widetilde{P}_{j}\right)=B_{1} \times$ $\left(c_{1}-d_{1}\right)+B_{2} \times\left(c_{2}-d_{2}\right)+\cdots+B_{n} \times\left(c_{n}-d_{n}\right)$ and $S\left(\widetilde{P}_{j} \ominus \widetilde{P}_{k}\right)=B_{1} \times\left(d_{1}-e_{1}\right)+B_{2} \times\left(d_{2}-e_{2}\right)+\cdots+$ $B_{n} \times\left(d_{n}-e_{n}\right)$. Hence, $S\left(\widetilde{P}_{i} \ominus \widetilde{P}_{j}\right)+S\left(\widetilde{P}_{j} \ominus \widetilde{P}_{k}\right)=$ $\left(B_{1} \times\left(c_{1}-d_{1}\right)+B_{2} \times\left(c_{2}-d_{2}\right)+\cdots+B_{n} \times\left(c_{n}-\right.\right.$ $\left.\left.d_{n}\right)\right)+\left(B_{1} \times\left(d_{1}-e_{1}\right)+B_{2} \times\left(d_{2}-e_{2}\right)+\cdots+B_{n} \times\right.$ $\left.\left(d_{n}-e_{n}\right)\right)=\left(B_{1} \times\left(c_{1}-d_{1}\right)+B_{1} \times\left(d_{1}-e_{1}\right)\right)+$ $\left(B_{2} \times\left(c_{2}-d_{2}\right)+B_{2} \times\left(d_{2}-e_{2}\right)\right)+\cdots+\left(B_{n} \times\left(c_{n}-\right.\right.$ $\left.\left.d_{n}\right)+B_{n} \times\left(d_{n}-e_{n}\right)\right)=B_{1} \times\left(c_{1}-e_{1}\right)+B_{2} \times\left(c_{2}-\right.$ $\left.e_{2}\right)+\cdots+B_{n} \times\left(c_{n}-e_{n}\right)=S\left(\widetilde{P}_{i} \ominus \widetilde{P}_{k}\right)$. So $S\left(\widetilde{P}_{i} \ominus\right.$ $\left.\widetilde{P}_{k}\right)=S\left(\widetilde{P}_{i} \ominus \widetilde{P}_{j}\right)+S\left(\widetilde{P}_{j} \ominus \widetilde{P}_{k}\right) \leqslant 0$, hence, $\widetilde{P}_{i} \leqslant s \widetilde{P}_{k}$.

Thus " $\leqslant s$ " is a pre-order relation of fuzzy sets.

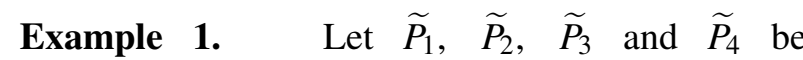
four evaluating values of the alternatives $x_{1}, x_{2}, x_{3}$ and $x_{4}$, which are fuzzy sets on $S$, i.e., $\widetilde{P}_{1}=$ $0.045 / s_{-1}+0.165 / s_{0}+0.4 / s_{1}+0.25 / s_{2}+0.14 / s_{3}$, $\widetilde{P}_{2}=0.14 / s_{0}+0.17 / s_{1}+0.315 / s_{2}+0.285 / s_{3}+$ $0.09 / s_{4}, \widetilde{P}_{3}=0.08 / s_{-2}+0.06 / s_{-1}+0.225 / s_{0}+$ $0.35 / s_{1}+0.14 / s_{2}+0.1 / s_{3}+0.045 / s_{4}$ and $\widetilde{P}_{4}=$ $0.15 / s_{0}+0.15 / s_{1}+0.36 / s_{2}+0.215 / s_{3}+0.125 / s_{4}$. 
According to Eq.(4), we have

$$
\begin{aligned}
& S\left(\widetilde{P}_{1} \ominus \widetilde{P}_{1}\right)=0, S\left(\widetilde{P}_{1} \ominus \widetilde{P}_{2}\right)=-0.74, \\
& S\left(\widetilde{P}_{1} \ominus \widetilde{P}_{3}\right)=0.37, S\left(\widetilde{P}_{1} \ominus \widetilde{P}_{4}\right)=-0.74 .
\end{aligned}
$$

Clearly, $S\left(\widetilde{P}_{1} \ominus \widetilde{P}_{2}\right)=S\left(\widetilde{P}_{1} \ominus \widetilde{P}_{4}\right)<S\left(\widetilde{P}_{1} \ominus \widetilde{P}_{1}\right)<$ $S\left(\widetilde{P}_{1} \ominus \widetilde{P}_{3}\right)$. In this example, we can see that $\widetilde{P}_{2} \leqslant{ }_{s} \widetilde{P}_{4}$ and $\widetilde{P}_{4} \leqslant_{s} \widetilde{P}_{2}$, but $\widetilde{P}_{2} \neq \widetilde{P}_{4}$. So, " $\leqslant_{s}$ " is not an partial order relation of fuzzy sets. So, we can not select the most desirable one from the alternatives $x_{2}$ and $x_{4}$.

\section{An Improved Ranking Strategy}

In this section, in order to solve the above problem, we provide an improved ranking strategy.

Definition 5. Let $\widetilde{P}=a_{1} / s_{B_{1}}+a_{2} / s_{B_{2}}+\cdots+a_{n} / s_{B_{n}}$ be a fuzzy set, where $B_{1}, B_{2}, \cdots, B_{n}$ are integers, $B_{1}<B_{2}<\cdots<B_{n}, s_{B_{1}}<s_{B_{2}}<\cdots<s_{B_{n}}$, $a_{i}$ denotes the grade of membership of $s_{B_{i}}$ in the fuzzy set $\widetilde{P}, a_{i} \in[0,1], 1 \leqslant i \leqslant n$. Then, the expectation of the fuzzy set $\widetilde{P}$ is defined by

$$
E(\widetilde{P})=a_{1} \times B_{1}+a_{2} \times B_{2}+\cdots+a_{n} \times B_{n} .
$$

For any fuzzy set $\widetilde{P}_{i}=c_{1} / s_{B_{1}}+c_{2} / s_{B_{2}}+\cdots+$ $c_{n} / s_{B_{n}}$ and $\widetilde{P}_{j}=d_{1} / s_{B_{1}}+d_{2} / s_{B_{2}}+\cdots+d_{n} / s_{B_{n}}$ on $U=\left\{s_{B_{1}}, s_{B_{2}}, \cdots, s_{B_{n}}\right\}$, obviously $E\left(\widetilde{P}_{i}\right)=E\left(\widetilde{P}_{j}\right)$ if and only if $S\left(\widetilde{P}_{i} \ominus \widetilde{P}_{j}\right)=0$.

Example 2. Let $\widetilde{P}_{1}$ and $\widetilde{P}_{2}$ be two evaluating values of the alternatives $x_{1}$ and $x_{2}$, which are fuzzy sets on $S$, i.e., $\widetilde{P}_{1}=0.035 / s_{-1}+0.165 / s_{0}+0.45 / s_{1}+$ $0.25 / s_{2}+0.1 / s_{3}$ and $\widetilde{P}_{2}=0.38 / s_{0}+0.315 / s_{1}+$ $0.2 / s_{2}+0.1 / s_{3}+0.05 / s_{4}$.

According to Eq.(4), we have

$S\left(\widetilde{P}_{1} \ominus \widetilde{P}_{2}\right)=\left(0.035 / s_{-1}+0.165 / s_{0}+0.45 / s_{1}+\right.$ $\left.0.25 / s_{2}+0.1 / s_{3}\right) \ominus\left(0.38 / s_{0}+0.315 / s_{1}+0.2 / s_{2}+\right.$ $\left.0.1 / s_{3}+0.05 / s_{4}\right)=(-1) \times(0.035-0)+0 \times$ $(0.165-0.38)+1 \times(0.45-0.315)+2 \times(0.25-$ $0.2)+3 \times(0.1-0.1)+4 \times(0-0.05)=0$.

According to Eq.(5), we have

$E\left(\widetilde{P}_{1}\right)=0.035 \times(-1)+0.165 \times 0+0.45 \times 1+$ $0.25 \times 2+0.1 \times 3=1.215, E\left(\widetilde{P}_{2}\right)=0.38 \times 0+$
$0.315 \times 1+0.2 \times 2+0.1 \times 3+0.05 \times 4=1.215$, $E\left(\widetilde{P}_{1}\right)=E\left(\widetilde{P}_{2}\right)$.

Definition 6. Let $\widetilde{P}=a_{1} / s_{B_{1}}+a_{2} / s_{B_{2}}+\cdots+a_{n} / s_{B_{n}}$ be a fuzzy set, where $B_{1}, B_{2}, \cdots, B_{n}$ are integers, $B_{1}<B_{2}<\cdots<B_{n}, s_{B_{1}}<s_{B_{2}}<\cdots<s_{B_{n}}, a_{i}$ denotes the grade of membership of $s_{B_{i}}$ in the fuzzy set $\widetilde{P}$, $a_{i} \in[0,1], 1 \leqslant i \leqslant n$. Then, the k-order deviation of the fuzzy set $\widetilde{P}$ is defined by

$$
\begin{aligned}
D^{k}(\widetilde{P})= & a_{1} \times\left(B_{1}-E(\widetilde{P})\right)^{k}+a_{2} \times\left(B_{2}-E(\widetilde{P})\right)^{k} \\
& +\cdots+a_{n} \times\left(B_{n}-E(\widetilde{P})\right)^{k},
\end{aligned}
$$

where $k$ is a positive integer, $k \in N_{+}$.

Theorem 2. For any fuzzy set $\widetilde{P}_{i}=a_{1} / s_{B_{1}}+$ $a_{2} / s_{B_{2}}+\cdots+a_{n} / s_{B_{n}}, \quad \widetilde{P}_{j}=b_{1} / s_{B_{1}}+b_{2} / s_{B_{2}}+$ $\cdots+b_{n} / s_{B_{n}}$ on $U=\left\{s_{B_{1}}, s_{B_{2}}, \cdots, s_{B_{n}}\right\}$, where $B_{1}, B_{2}, \cdots, B_{n}$ are integers, $B_{1}<B_{2}<\cdots<B_{n}$, $s_{B_{1}}<s_{B_{2}}<\cdots<s_{B_{n}}, E\left(\widetilde{P}_{i}\right)=E\left(\widetilde{P}_{j}\right)$. If $E\left(\widetilde{P}_{i}\right) \neq B_{k}$, for all $k \in\{1,2, \cdots, n\}$, then $\widetilde{P}_{i}=\widetilde{P}_{j}$ if and only if for any $k, D^{k}\left(\widetilde{P}_{i}\right)=D^{k}\left(\widetilde{P}_{j}\right)$, where $k \in\{1,2, \cdots, n\}$.

Proof. " $\Longrightarrow$ " Obviously.

“ " Suppose $D^{k}\left(\widetilde{P}_{i}\right)=D^{k}\left(\widetilde{P}_{j}\right)$ for all $k \in$ $\{1,2, \cdots, n\}$, let $E=E\left(\widetilde{P}_{i}\right), x_{m}=a_{m}-b_{m}$ for all $m \in\{1,2, \cdots, n\}$, i.e.,

$$
\begin{cases}\left(B_{1}-E\right) \times x_{1}+ & \cdots+\left(B_{n}-E\right) \times x_{n}=0, \\ \left(B_{1}-E\right)^{2} \times x_{1}+ & \cdots+\left(B_{n}-E\right)^{2} \times x_{n}=0, \\ & \cdots \\ \left(B_{1}-E\right)^{n} \times x_{1}+ & \cdots+\left(B_{n}-E\right)^{n} \times x_{n}=0 .\end{cases}
$$

We see that it is a homogeneous system of linear equations, where $\left(a_{1}-b_{1}\right), \cdots,\left(a_{n}-b_{n}\right)$ are unknown. The coefficient matrix is

$$
A=\left(\begin{array}{cccc}
\left(B_{1}-E\right) & \left(B_{2}-E\right) & \cdots & \left(B_{n}-E\right) \\
\left(B_{1}-E\right)^{2} & \left(B_{2}-E\right)^{2} & \cdots & \left(B_{n}-E\right)^{2} \\
\vdots & \vdots & \ddots & \vdots \\
\left(B_{1}-E\right)^{n} & \left(B_{2}-E\right)^{n} & \cdots & \left(B_{n}-E\right)^{n}
\end{array}\right),
$$

we get

$$
\left|\begin{array}{cccc}
|A|=\left(B_{1}-E\right) \times \cdots \times\left(B_{n}-E\right) \times & \\
1 & 1 & \cdots & 1 \\
\left(B_{1}-E\right) & \left(B_{2}-E\right) & \cdots & \left(B_{n}-E\right) \\
\vdots & \vdots & \ddots & \vdots \\
\left(B_{1}-E\right)^{(n-1)} & \left(B_{2}-E\right)^{(n-1)} & \cdots & \left(B_{n}-E\right)^{(n-1)}
\end{array}\right| .
$$


Clearly, the right determinant is a vandermonde determinant, so

$$
\begin{aligned}
|A|= & \left(B_{1}-E\right) \times \cdots \times\left(B_{n}-E\right) \times \\
& \prod_{1 \leqslant j<i \leqslant n}\left(\left(B_{i}-E\right)-\left(B_{j}-E\right)\right) .
\end{aligned}
$$

Because of $E \neq B_{k}$, we get $\left(B_{k}-E\right) \neq 0$, for all $k \in\{1,2, \cdots, n\}$, and $\left(B_{i}-E\right) \neq\left(B_{j}-E\right)$, for any $i, j \in\{1,2, \cdots, n\}$. So, $|A| \neq 0$. Thus Eq.(7) has only null solution, we get $\left(a_{l}-b_{l}\right)=0$ for $l=1$ to $n$. So, $\widetilde{P}_{i}=\widetilde{P}_{j}$.

Definition 7. Let $\widetilde{P}_{1}=a_{1} / s_{B_{1}}+a_{2} / s_{B_{2}}+\cdots+$ $a_{n} / s_{B_{n}}, \widetilde{P}_{2}=b_{1} / s_{B_{1}}+b_{2} / s_{B_{2}}+\cdots+b_{n} / s_{B_{n}}$ be two fuzzy sets in the universe of discourse $U$, where $U=\left\{s_{B_{1}}, s_{B_{2}}, \cdots, s_{B_{n}}\right\}$, where $B_{1}, B_{2}, \cdots, B_{n}$ are integers, $B_{1}<B_{2}<\cdots<B_{n}, s_{B_{1}}<s_{B_{2}}<\cdots<s_{B_{n}}$, and $E\left(\widetilde{P}_{1}\right)=E\left(\widetilde{P}_{2}\right)$. (1) Assume $E\left(\widetilde{P}_{1}\right) \neq B_{i}$, for all $i \in$ $\{1,2, \cdots, n\}$, if $D^{k}\left(\widetilde{P}_{1}\right)<D^{k}\left(\widetilde{P}_{2}\right)$, where $k$ is the minimum positive integer such that $D^{k}\left(\widetilde{P}_{1}\right) \neq D^{k}\left(\widetilde{P}_{2}\right)$, then $\widetilde{P}_{1}>\widetilde{P}_{2}$. (2) Assume $E\left(\widetilde{P}_{1}\right)=B_{i}$, where $i$ is an integer and $i \in\{1,2, \cdots, n\}$, if $a_{i}>b_{i}$, then $\widetilde{P}_{1}>\widetilde{P}_{2}$, if $a_{i}<b_{i}$, then $\widetilde{P}_{1}<\widetilde{P}_{2}$, if $a_{i}=b_{i}$, it is similar to (1).

Example 3. (1) Let $\widetilde{P}_{1}$ and $\widetilde{P}_{2}$ be two evaluating values of the alternatives $x_{1}$ and $x_{2}$, which are fuzzy sets on $S$, i.e., $\widetilde{P}_{1}=0.035 / s_{-1}+0.165 / s_{0}+0.45 / s_{1}+$ $0.25 / s_{2}+0.1 / s_{3}$ and $\widetilde{P}_{2}=0.38 / s_{0}+0.315 / s_{1}+$ $0.2 / s_{2}+0.1 / s_{3}+0.05 / s_{4}$.

According to Eq.(5), we have $E\left(\widetilde{P}_{1}\right)=E\left(\widetilde{P}_{2}\right)=$ 1.215. So, calculate the k-order deviation $D^{k}\left(\widetilde{P}_{1}\right)$ and $D^{k}\left(\widetilde{P}_{2}\right)$ of $\widetilde{P}_{1}$ and $\widetilde{P}_{2}$, get $D^{1}\left(\widetilde{P}_{1}\right)=D^{1}\left(\widetilde{P}_{2}\right)=0$, $D^{2}\left(\widetilde{P}_{1}\right)=0.90877501, D^{2}\left(\widetilde{P}_{2}\right)=1.40520513$, since $D^{2}\left(\widetilde{P}_{1}\right)<D^{2}\left(\widetilde{P}_{2}\right)$, we get $\widetilde{P}_{1}>\widetilde{P}_{2}$.

(2) Let $\widetilde{P}_{3}$ and $\widetilde{P}_{4}$ be two evaluating values of the alternatives $x_{3}$ and $x_{4}$, which are fuzzy sets on $S$, i.e., $\widetilde{P}_{3}=0.2 / s_{-1}+0.15 / s_{0}+0.3 / s_{1}+0.2 / s_{2}+0.1 / s_{3}+$ $0.05 / s_{4}$ and $\widetilde{P}_{4}=0.2 / s_{-1}+0.05 / s_{0}+0.4 / s_{1}+$ $0.25 / s_{2}+0.1 / s_{3}$.

According to Eq.(5), we have $E\left(\widetilde{P}_{3}\right)=E\left(\widetilde{P}_{4}\right)=$ 1 . Since $0.3<0.4$, we get $\widetilde{P}_{3}<\widetilde{P}_{4}$.

Theorem 3. Let $\widetilde{P}_{1}, \widetilde{P}_{2}$ and $\widetilde{P}_{3}$ be three fuzzy sets in the universe of discourse $U$, where $U=$ $\left\{s_{B_{1}}, s_{B_{2}}, \cdots, s_{B_{n}}\right\}$, where $U=\left\{s_{B_{1}}, s_{B_{2}}, \cdots, s_{B_{n}}\right\}$, where $B_{1}, B_{2}, \cdots, B_{n}$ are integers, $B_{1}<B_{2}<\cdots<$
$B_{n}, s_{B_{1}}<s_{B_{2}}<\cdots<s_{B_{n}}$. Assume $E\left(\widetilde{P}_{1}\right)=E\left(\widetilde{P}_{2}\right)=$ $E\left(\widetilde{P}_{3}\right)$. If $\widetilde{P}_{1}>\widetilde{P}_{2}$ and $\widetilde{P}_{2}>\widetilde{P}_{3}$, then $\widetilde{P}_{1}>\widetilde{P}_{3}$.

Proof. (1) Suppose $E\left(\widetilde{P}_{1}\right) \neq B_{i}$, for all $i \in$ $\{1,2, \cdots, n\}$, since $\widetilde{P}_{1}>\widetilde{P}_{2}$, so $D^{k_{1}}\left(\widetilde{P}_{1}\right)<D^{k_{1}}\left(\widetilde{P}_{2}\right)$, where $k_{1}$ is the minimum positive integer such that $D^{k_{1}}\left(\widetilde{P}_{1}\right) \neq D^{k_{1}}\left(\widetilde{P}_{2}\right)$. Since $\widetilde{P}_{2}>\widetilde{P}_{3}$, so $D^{k_{2}}\left(\widetilde{P}_{2}\right)<$ $D^{k_{2}}\left(\widetilde{P}_{3}\right)$, where $k_{2}$ is the minimum positive integer such that $D^{k_{2}}\left(\widetilde{P}_{2}\right) \neq D^{k_{2}}\left(\widetilde{P}_{3}\right)$. If $k_{2}>k_{1}, D^{k_{1}}\left(\widetilde{P}_{1}\right)<$ $D^{k_{1}}\left(\widetilde{P}_{2}\right)=D^{k_{1}}\left(\widetilde{P}_{3}\right)$, so $D^{k_{1}}\left(\widetilde{P}_{1}\right)<D^{k_{1}}\left(\widetilde{P}_{3}\right)$ and $k_{1}$ is the minimum positive integer such that $D^{k_{1}}\left(\widetilde{P}_{1}\right) \neq$ $D^{k_{1}}\left(\widetilde{P}_{3}\right)$, then $\widetilde{P}_{1}>\widetilde{P}_{3}$. If $k_{2}=k_{1}, D^{k_{1}}\left(\widetilde{P}_{1}\right)<$ $D^{k_{1}}\left(\widetilde{P}_{2}\right)<D^{k_{1}}\left(\widetilde{P}_{3}\right)$, so $D^{k_{1}}\left(\widetilde{P}_{1}\right)<D^{k_{1}}\left(\widetilde{P}_{3}\right)$ and $k_{1}$ is the minimum positive integer such that $D^{k_{1}}\left(\widetilde{P}_{1}\right) \neq$ $D^{k_{1}}\left(\widetilde{P}_{3}\right)$, then $\widetilde{P}_{1}>\widetilde{P}_{3}$. If $k_{2}<k_{1}, D^{k 2}\left(\widetilde{P}_{1}\right)=$ $D^{k_{2}}\left(\widetilde{P}_{2}\right)<D^{k_{2}}\left(\widetilde{P}_{3}\right)$, so $D^{k_{2}}\left(\widetilde{P}_{1}\right)<D^{k_{2}}\left(\widetilde{P}_{3}\right)$ and $k_{2}$ is the minimum positive integer such that $D^{k_{2}}\left(\widetilde{P}_{1}\right) \neq$ $D^{k_{2}}\left(\widetilde{P}_{3}\right)$, then $\widetilde{P}_{1}>\widetilde{P}_{3}$.

(2) Suppose $E\left(\widetilde{P}_{1}\right)=B_{i}, i$ is an integer and $i \in$ $\{1,2, \cdots, n\}$, if $a_{i} \neq b_{i}$, it is obvious, if $a_{i}=b_{i}$, it is similar to (1).

\section{The Improved Method for Fuzzy Multiple Attribute Group Decision Making}

In this section, first, we propose an improved method for fuzzy multiple attribute group decision making. Then, compare the improved method with Chen's method ${ }^{2}$.

The improved method: Assume that there are $n$ alternatives $x_{1}, x_{2}, \cdots, x_{n}$ and $m$ attributes $f_{1}, f_{2}, \cdots, f_{m}$. Assume that there are $g$ decision makers $D_{1}, D_{2}, \cdots, D_{g}$. Let $H=\left[h_{1}, h_{2}, \cdots, h_{g}\right]^{T}$ be the weighting vector of the decision makers, where $h_{i}$ denotes the weight of the decision maker $D_{i}, 1 \leqslant$ $i \leqslant g$ and $\sum_{i=1}^{g} h_{i}=1$. Let $V=\left[v_{1}, v_{2}, \cdots, v_{m}\right]^{T}$ be the weighting vector of the attributes, where $v_{i}$ denotes the weight of the attribute $f_{i}, 1 \leqslant i \leqslant m$ and $\sum_{i=1}^{m} v_{i}=1$. The proposed algorithm for fuzzy group decision making is now presented as follows:

Step 1: Construct the fuzzy evaluating matrix $\widetilde{F}_{k}$ for the decision maker $D_{k}$ with respect to the at- 
tribute $f_{i}$ of the alternative $x_{j}$, shown as follows:

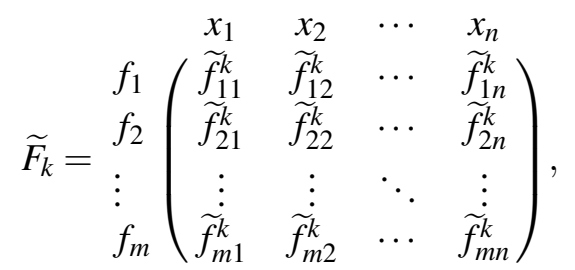

where, $1 \leqslant i \leqslant m, 1 \leqslant j \leqslant n, 1 \leqslant k \leqslant g$ and $\widetilde{f}_{i j}^{k}$ is a fuzzy set.

Step 2: Assume that the weighting vector $W=$ $\left[w_{1}, w_{2}, \cdots, w_{k}\right]^{T}$. Based on the weighting vector $W$ and the fuzzy evaluating matrix $\widetilde{F}_{k}$, calculate the weighted value $\widetilde{Z}_{i j}$ of the attribute $f_{i}$ of the alternative $x_{j}$ by using the FIOWA operators, where

$$
\begin{aligned}
\widetilde{Z}_{i j} & =F_{F I O W A}\left(\left\langle h_{1}, \widetilde{f}_{i j}^{1}\right\rangle,\left\langle h_{2}, \widetilde{f}_{i j}^{2}\right\rangle, \cdots,\left\langle h_{k}, \widetilde{f}_{i j}^{k}\right\rangle\right) \\
& =w_{1} \widetilde{f}_{i j}^{1} \oplus w_{2} \widetilde{f}_{i j}^{2} \oplus \cdots \oplus w_{k} \widetilde{f}_{i j}^{k}
\end{aligned}
$$

$1 \leqslant i \leqslant m, 1 \leqslant j \leqslant n, 1 \leqslant k \leqslant g, h_{k}$ denotes the weighting vector of the decision maker $D_{k} . \widetilde{f}_{i j}^{k}$ is the value of the OWA pair having the $k$ th largest order inducing $h$ value.

Step 3: Assume that the weighting vector $R=$ $\left[r_{1}, r_{2}, \cdots, r_{i}\right]^{T}$. Based on the weighting vector $R$ and the weighted value $\widetilde{Z}_{i j}$, calculate the score $\widetilde{E}_{j}$ of the alternative $x_{j}$, and we get

$$
\begin{aligned}
\widetilde{E}_{j} & =F_{F I O W A}\left(\left\langle v_{1}, \widetilde{Z}_{1 j}\right\rangle,\left\langle v_{2}, \widetilde{Z}_{2 j}\right\rangle, \cdots,\left\langle v_{i}, \widetilde{Z}_{i j}\right\rangle\right) \\
& =r_{1} \widetilde{Z}_{1 j}^{\prime} \oplus r_{2} \widetilde{Z}_{2 j}^{\prime} \oplus \cdots \oplus r_{i} \widetilde{Z}_{i j}^{\prime},
\end{aligned}
$$

where $1 \leqslant i \leqslant m, 1 \leqslant j \leqslant n$. $v_{i}$ denotes the weight of the attribute $f_{i}$. $\widetilde{Z}_{i j}^{\prime}$ is the value of the OWA pair having the $i$ th largest order inducing $v$ value.

Step 4: Based on Eq.(5), calculate the score $E\left(\widetilde{E}_{i}\right)$ of $\widetilde{E}_{i}$, where $1 \leqslant i \leqslant n$.

Step 5: Sort the values of $E\left(\widetilde{E}_{1}\right), E\left(\widetilde{E}_{2}\right), \cdots$, and $E\left(\widetilde{E}_{n}\right)$ in a descending sequence. Let $T=$ $\left\{\widetilde{E}_{i} \mid E\left(\widetilde{E}_{i}\right) \geqslant E\left(\widetilde{E}_{j}\right), j \neq i, 1 \leqslant i, j \leqslant n\right\}$. If $|T|=1$, the most desirable alternative is $x_{i}$. If $|T| \geqslant 2$, based on definition 6, calculate the k-order deviation $D^{k}\left(\widetilde{E}_{j}\right)$ of $\widetilde{E}_{j}$, where $\widetilde{E}_{j} \in T$, and based on definition 7 , select the desirable $\widetilde{E}_{k}$, thus the best alternative is $x_{k}$.

Step 1-3 are the same as Chen's method ${ }^{2}$, the ranking strategy of Chen's method ${ }^{2}$ is to calculate the score $S\left(\widetilde{E}_{1} \ominus \widetilde{E}_{j}\right)$ of the weighted difference of membership values between $\widetilde{E}_{1}$ and $\widetilde{E}_{j}$, where $1 \leqslant$ $j \leqslant n$. Then based on definition 4 select the best $\widetilde{E}_{j}$, the corresponding $x_{j}$ is the best alternative, where $1 \leqslant j \leqslant n$. Let $B=\left\{\widetilde{E}_{i} \mid S\left(\widetilde{E}_{1} \ominus \widetilde{E}_{i}\right) \leqslant S\left(\widetilde{E}_{1} \ominus \widetilde{E}_{j}\right)\right), j \neq$ $i, 1 \leqslant i, j \leqslant n\}$, when $|B| \geqslant 2$, Chen's method can not choose the best alternative. So, in the previous section, we proposed an improved ranking strategy, it is an alternative method to solve this problem, the improved method is a more complete manner than Chen's method ${ }^{2}$.

\section{Numerical Example}

In this section, we use an example to illustrate the proposed method for handling fuzzy group decision making problems.

Example 4. Assume that there are five alternatives $x_{1}, x_{2}, x_{3}, x_{4}$ and $x_{5}$ and there are three decision makers $D_{1}, D_{2}$ and $D_{3}$ who want to choose the best alternative among $x_{1}, x_{2}, x_{3}, x_{4}$ and $x_{5}$. Assume that there are four attributes, i.e., the risk analysis (denoted by $f_{1}$ ), the growth analysis (denoted by $f_{2}$ ), the social-political impact analysis (denoted by $f_{3}$ ) and the environmental impact analysis (denoted by $f_{4}$ ). Assume that the weighting vector $H$ of the decision makers is shown as follows: $H=\left[h_{1}, h_{2}, h_{3}\right]^{T}=[0.5,0.3,0.2]^{T}$. Assume that the weighting vector $V$ of the four attributes is shown as follows: $V=\left[v_{1}, v_{2}, v_{3}, v_{4}\right]^{T}=[0.3,0.4,0.2,0.1]^{T}$. Assume that the fuzzy evaluating values of the alternatives given by the decision makers with respect to different attributes are as follows:

Step 1: Construct the fuzzy evaluating matrix $\widetilde{F}_{k}$ for the decision maker $D_{k}$ with respect to the attribute $f_{i}$ of the alternative $x_{j}$, where $1 \leqslant k \leqslant 3$, $1 \leqslant i \leqslant 4,1 \leqslant j \leqslant 5$ shown as follows:

$$
\widetilde{F}_{1}=\begin{gathered}
f_{1} \\
f_{2} \\
f_{3} \\
f_{4}
\end{gathered}\left(\begin{array}{ccccc}
x_{1} & x_{2} & x_{3} & x_{4} & x_{5} \\
\widetilde{f}_{11}^{1} & \widetilde{f}_{12}^{1} & \widetilde{f}_{13}^{1} & \widetilde{f}_{14}^{1} & \widetilde{f}_{15}^{1} \\
\widetilde{f}_{31}^{1} & \widetilde{f}_{32}^{1} & \widetilde{f}_{33}^{1} & \widetilde{f}_{34}^{1} & \widetilde{f}_{25}^{1} \\
\widetilde{f}_{41}^{1} & \widetilde{f}_{42}^{1} & \widetilde{f}_{43}^{1} & \widetilde{f}_{44}^{1} & \widetilde{f}_{45}^{1}
\end{array}\right),
$$




$$
\begin{aligned}
& \widetilde{F}_{2}=\begin{array}{c}
f_{1} \\
f_{2} \\
f_{3} \\
f_{4}
\end{array}\left(\begin{array}{ccccc}
x_{1} & x_{2} & x_{31} & x_{4} & x_{5} \\
\widetilde{f}_{11}^{2} & \widetilde{f}_{12}^{2} & \widetilde{f}_{13}^{2} & \widetilde{f}_{14}^{2} & \widetilde{f}_{15}^{2} \\
\widetilde{f}_{31}^{2} & \widetilde{f}_{32}^{2} & \widetilde{f}_{23}^{2} & \widetilde{f}_{24}^{2} & \widetilde{f}_{25}^{2} \\
\widetilde{f}_{41}^{2} & \widetilde{f}_{42}^{2} & \widetilde{f}_{43}^{2} & \widetilde{f}_{44}^{2} & \widetilde{f}_{45}^{2}
\end{array}\right),
\end{aligned}
$$

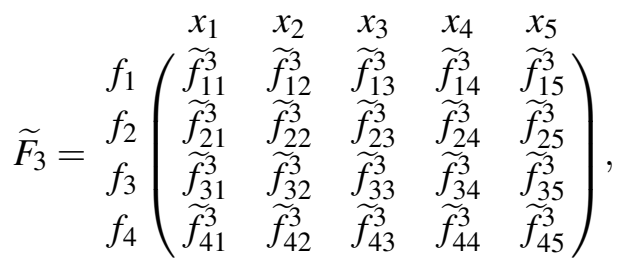

where $\widetilde{f}_{11}^{1}=0.5 / s_{1}+0.5 / s_{2}, \widetilde{f}_{12}^{1}=0.5 / s_{2}+0.5 / s_{3}$, $\widetilde{f}_{13}^{1}=0.5 / s_{-1}+0.5 / s_{0}, \quad \widetilde{f}_{14}^{1}=0.5 / s_{2}+0.5 / s_{3}$, $\widetilde{f}_{15}^{1}=0.5 / s_{0}+0.5 / s_{1}+0.5 / s_{2}, \quad \widetilde{f}_{21}^{1}=0.5 / s_{1}+$ $0.5 / s_{3}, \quad \widetilde{f}_{22}^{1}=0.5 / s_{0}+0.5 / s_{1}, \quad \widetilde{f}_{23}^{1}=0.5 / s_{-2}+$ $0.5 / s_{1}, \quad \widetilde{f}_{24}^{1}=0.5 / s_{0}+0.5 / s_{1}, \quad \widetilde{f}_{25}^{1}=0.5 / s_{3}+$ $0.25 / s_{4}, \quad \widetilde{f}_{31}^{1}=0.5 / s_{2}+0.5 / s_{3}, \quad \widetilde{f}_{32}^{1}=0.5 / s_{0}+$ $0.5 / s_{2}, \quad \widetilde{f}_{33}^{1}=0.5 / s_{1}+0.5 / s_{3}, \quad \widetilde{f}_{34}^{1}=0.5 / s_{-1}+$ $0.5 / s_{0}, \widetilde{f}_{35}^{1}=0.5 / s_{2}+0.5 / s_{3}, \widetilde{f}_{41}^{1}=0.5 / s_{1}+0.5 / s_{3}$, $\widetilde{f}_{42}^{1}=0.5 / s_{0}+0.5 / s_{2}, \widetilde{f}_{43}^{1}=0.5 / s_{1}+0.5 / s_{2}, \widetilde{f}_{44}^{1}=$ $0.5 / s_{3}+0.5 / s_{4}, \quad \widetilde{f}_{45}^{1}=0.5 / s_{2}, \quad \widetilde{f}_{11}^{2}=0.5 / s_{0}+$ $0.5 / s_{1}, \quad \widetilde{f}_{12}^{2}=0.5 / s_{3}+0.5 / s_{4}, \quad \widetilde{f}_{13}^{2}=0.5 / s_{0}+$ $0.5 / s_{1}, \widetilde{f}_{14}^{2}=0.5 / s_{1}+0.5 / s_{2}, \widetilde{f}_{15}^{2}=0.5 / s_{0}+0.5 / s_{2}$, $\widetilde{f}_{21}^{2}=0.5 / s_{1}+0.5 / s_{2}, \widetilde{f}_{22}^{2}=0.5 / s_{2}+0.5 / s_{3}, \widetilde{f}_{23}^{2}=$ $0.5 / s_{0}+0.5 / s_{1}, \quad \widetilde{f}_{24}^{2}=0.5 / s_{-1}+0.5 / s_{1}, \quad \widetilde{f}_{25}^{2}=$ $0.5 / s_{2}, \quad \widetilde{f}_{31}^{2}=0.5 / s_{0}+0.5 / s_{1}, \quad \widetilde{f}_{32}^{2}=0.5 / s_{1}+$ $0.5 / s_{2}, \quad \widetilde{f}_{33}^{2}=0.5 / s_{2}+0.5 / s_{3}, \quad \widetilde{f}_{34}^{2}=0.5 / s_{0}+$ $0.5 / s_{2}, \quad \widetilde{f}_{35}^{2}=0.5 / s_{1}+0.5 / s_{3}, \quad \widetilde{f}_{41}^{2}=0.5 / s_{0}+$ $0.5 / s_{2}, \quad \widetilde{f}_{42}^{2}=0.5 / s_{1}+0.5 / s_{2}, \quad \widetilde{f}_{43}^{2}=0.5 / s_{2}+$ $0.5 / s_{3}, \quad \widetilde{f}_{44}^{2}=0.5 / s_{2}+0.5 / s_{3}, \quad \widetilde{f}_{45}^{2}=0.5 / s_{1}+$ $0.5 / s_{2} \quad \widetilde{f}_{11}^{3}=0.5 / s_{-1}+0.5 / s_{0}, \quad \widetilde{f}_{12}^{3}=0.5 / s_{3}+$ $0.5 / s_{4}, \widetilde{f}_{13}^{3}=0.5 / s_{1}+0.5 / s_{2}, \widetilde{f}_{14}^{3}=0.5 / s_{3}+0.5 / s_{4}$, $\widetilde{f}_{15}^{3}=0.5 / s_{0}+0.5 / s_{1}+0.5 / s_{2}, \quad \widetilde{f}_{21}^{3}=0.5 / s_{1}+$ $0.5 / s_{2}, \quad \widetilde{f}_{22}^{3}=0.5 / s_{2}+0.2 / s_{3}, \quad \widetilde{f}_{23}^{3}=0.5 / s_{0}+$ $0.5 / s_{1}, \quad \widetilde{f}_{24}^{3}=0.5 / s_{1}+0.5 / s_{3}, \quad \widetilde{f}_{25}^{3}=0.5 / s_{2}+$ $0.5 / s_{3}, \quad \widetilde{f}_{31}^{3}=0.5 / s_{0}+0.5 / s_{1}, \quad \widetilde{f}_{32}^{3}=0.5 / s_{1}+$ $0.5 / s_{2}, \quad \widetilde{f}_{33}^{3}=0.5 / s_{2}+0.5 / s_{4}, \quad \widetilde{f}_{34}^{3}=0.5 / s_{1}+$ $0.5 / s_{2}, \widetilde{f}_{35}^{3}=0.5 / s_{3}+0.5 / s_{4}, \widetilde{f}_{41}^{3}=0.5 / s_{1}+0.5 / s_{2}$, $\widetilde{f}_{42}^{3}=0.5 / s_{1}+0.5 / s_{3}, \widetilde{f}_{43}^{3}=0.5 / s_{3}+0.5 / s_{4}, \widetilde{f}_{44}^{3}=$ $0.5 / s_{0}+0.5 / s_{2}, \widetilde{f}_{45}^{3}=0.5 / s_{2}+0.5 / s_{3}+0.5 / s_{4}$.

Step 2: Assume that the weighting vector $W=$ $\left[w_{1}, w_{2}, w_{3}\right]^{T}=[0.4,0.3,0.3]^{T}$. Based on Eq.(8), we get the value $\widetilde{Z}_{i j}$ by using the FIOWA operators shown as follows:

$$
\begin{aligned}
\widetilde{Z}_{i j} & =F_{\text {FIOWA }}\left(\left\langle h_{1}, \widetilde{f}_{i j}^{1}\right\rangle,\left\langle h_{2}, \widetilde{f}_{i j}^{2}\right\rangle,\left\langle h_{3}, \widetilde{f}_{i j}^{3}\right\rangle\right) \\
& =w_{1} \widetilde{f}_{i j}^{1} \oplus w_{2} \widetilde{f}_{i j}^{2} \oplus w_{3} \widetilde{f}_{i j}^{3},
\end{aligned}
$$

where $\widetilde{f}_{i j}^{k}$ is the value of the OWA pair having the $k$ th largest order inducing $h$ value, $1 \leqslant i \leqslant 4,1 \leqslant j \leqslant 5$, $1 \leqslant k \leqslant 3$. The results are shown as follows:

$\widetilde{Z}_{11}=0.15 / s_{-1}+0.3 / s_{0}+0.35 / s_{1}+0.2 / s_{2}$, $\widetilde{Z}_{21}=0.5 / s_{1}+0.3 / s_{2}+0.2 / s_{3}, \quad \widetilde{Z}_{31}=0.3 / s_{0}+$ $0.3 / s_{1}+0.2 / s_{2}+0.2 / s_{3}, \widetilde{Z}_{41}=0.15 / s_{0}+0.35 / s_{1}+$ $0.3 / s_{2}+0.2 / s_{3}, \widetilde{Z}_{12}=0.2 / s_{2}+0.5 / s_{3}+0.3 / s_{4}$, $\widetilde{Z}_{22}=0.2 / s_{0}+0.2 / s_{1}+0.3 / s_{2}+0.3 / s_{3}, \widetilde{Z}_{32}=$ $0.2 / s_{0}+0.3 / s_{1}+0.5 / s_{2}, \widetilde{Z}_{42}=0.2 / s_{0}+0.3 / s_{1}+$ $0.35 / s_{2}+0.15 / s_{3}, \quad \widetilde{Z}_{13}=0.2 / s_{-1}+0.35 / s_{0}+$ $0.3 / s_{1}+0.15 / s_{2}, \quad \widetilde{Z}_{23}=0.2 / s_{-2}+0.3 / s_{0}+$ $0.5 / s_{1}, \widetilde{Z}_{33}=0.2 / s_{1}+0.3 / s_{2}+0.35 / s_{3}+0.15 / s_{4}$, $\widetilde{Z}_{43}=0.2 / s_{1}+0.35 / s_{2}+0.3 / s_{3}+0.15 / s_{4}, \widetilde{Z}_{14}=$ $0.15 / s_{1}+0.35 / s_{-1}+0.35 / s_{3}+0.15 / s_{4}, \quad \widetilde{Z}_{24}=$ $0.15 / s_{-1}+0.2 / s_{0}+0.5 / s_{1}+0.15 / s_{3}, \quad \widetilde{Z}_{34}=$ $0.2 / s_{-1}+0.35 / s_{0}+0.15 / s_{1}+0.3 / s_{2}, \quad \widetilde{Z}_{44}=$ $0.15 / s_{0}+0.3 / s_{2}+0.35 / s_{3}+0.2 / s_{4}, \widetilde{Z}_{15}=0.5 / s_{0}+$ $0.35 / s_{1}+0.5 / s_{2}, \widetilde{Z}_{25}=0.3 / s_{2}+0.35 / s_{3}+0.1 / s_{4}$, $\widetilde{Z}_{35}=0.15 / s_{1}+0.2 / s_{2}+0.3 / s_{3}+0.35 / s_{4}, \widetilde{Z}_{45}=$ $0.15 / s_{1}+0.5 / s_{2}+0.15 / s_{3}+0.15 / s_{4}$.

Step 3: Assume that the weighting vector $R=\left[r_{1}, r_{2}, r_{3}, r_{4}\right]^{T}=[0.4,0.3,0.2,0.1]^{T}$, based on Eq.(9), we get the value $\widetilde{E}_{j}$ of the alternative $x_{j}$, by using the FIOWA operators, where

$$
\begin{aligned}
\widetilde{E}_{j} & =F_{F I O W A}\left(\left\langle v_{1}, \widetilde{Z}_{1 j}\right\rangle,\left\langle v_{2}, \widetilde{Z}_{2 j}\right\rangle,\left\langle v_{3}, \widetilde{Z}_{3 j}\right\rangle,\left\langle v_{4}, \widetilde{Z}_{4 j}\right\rangle\right) \\
& =r_{1} \widetilde{Z}_{1 j}^{\prime} \oplus r_{2} \widetilde{Z}_{2 j}^{\prime} \oplus r_{3} \widetilde{Z}_{3 j}^{\prime} \oplus r_{4} \widetilde{Z}_{4 j}^{\prime},
\end{aligned}
$$

$v_{i}$ denotes the weight of the attribute $f_{i}, \widetilde{Z}_{i j}^{\prime}$ is the value of the OWA pair having the $i$ th largest order inducing $v$ value, $1 \leqslant i \leqslant 4,1 \leqslant j \leqslant 5$. The results are shown as follows:

$\widetilde{E}_{1}=0.045 / s_{-1}+0.165 / s_{0}+0.4 / s_{1}+0.25 / s_{2}+$ $0.14 / s_{3}, \quad \widetilde{E}_{2}=0.14 / s_{0}+0.17 / s_{1}+0.315 / s_{2}+$ $0.285 / s_{3}+0.09 / s_{4}, \quad \widetilde{E}_{3}=0.08 / s_{-2}+0.06 / s_{-1}+$ $0.225 / s_{0}+0.35 / s_{1}+0.14 / s_{2}+0.1 / s_{3}+0.045 / s_{4}$, $\widetilde{E}_{4}=0.1 / s_{-1}+0.165 / s_{0}+0.275 / s_{1}+0.195 / s_{2}+$ $0.2 / s_{3}+0.065 / s_{4}, \quad \widetilde{E}_{5}=0.15 / s_{0}+0.15 / s_{1}+$ $0.36 / s_{2}+0.215 / s_{3}+0.125 / s_{4}$. 
Step 4: Based on Eq.(5), we can calculate the score $E\left(\widetilde{E}_{i}\right)$ of $\widetilde{E}_{i}$, where $1 \leqslant i \leqslant 5$. The results are shown as follows:

$$
\begin{aligned}
& E\left(\widetilde{E}_{1}\right)=1.275, E\left(\widetilde{E}_{2}\right)=2.015, E\left(\widetilde{E}_{3}\right)=0.89, \\
& E\left(\widetilde{E}_{4}\right)=1.425, E\left(\widetilde{E}_{5}\right)=2.015 .
\end{aligned}
$$

Step 5: Sort the values of $E\left(\widetilde{E}_{1}\right), \cdots$, and $E\left(\widetilde{E}_{5}\right)$ in a descending sequence. Because $E\left(\widetilde{E}_{2}\right)=$ $E\left(\widetilde{E}_{5}\right)>E\left(\widetilde{E}_{4}\right)>E\left(\widetilde{E}_{1}\right)>E\left(\widetilde{E}_{3}\right)$, so $T=\left\{\widetilde{E}_{2}, \widetilde{E}_{5}\right\}$. Based on definition 6 , calculate the 1-order deviation $D^{1}\left(\widetilde{E}_{2}\right)=D^{1}\left(\widetilde{E}_{5}\right)=0$ of $\widetilde{E}_{2}$ and $\widetilde{E}_{5}$, the 2order deviation $D^{2}\left(\widetilde{E}_{2}\right)=1.37477501$ and $D^{2}\left(\widetilde{E}_{5}\right)=$ 1.46477501 of $\widetilde{E}_{2}$ and $\widetilde{E}_{5}$. Since $D^{2}\left(\widetilde{E}_{2}\right)<D^{2}\left(\widetilde{E}_{5}\right)$, based on definition 7, we can see that $\widetilde{E}_{2}$ is more stable than $\widetilde{E}_{5}$. So, the alternative $x_{2}$ is the best choice among the alternatives $x_{1}, x_{2}, x_{3}, x_{4}$ and $x_{5}$.

\section{Conclusions}

In this paper, we have presented an improved ranking strategy for fuzzy multiple attribute group decision making (FMAGDM). First, we show Chen's method ${ }^{2}$ can not choose the best alternative, when $S\left(\widetilde{P}_{i} \ominus \widetilde{P}_{j}\right)=0$ and $\widetilde{P}_{i} \neq \widetilde{P}_{j}$. The reason is because of " $\leqslant s$ " is not an partial order relation of fuzzy sets. Then, we propose an improved ranking strategy. We define the k-order deviation of the fuzzy sets, and prove two important theorems. Then, we propose an improved method for FMAGDM, which is a more complete manner than Chen's method ${ }^{2}$. This method is simple and easy to understand. The improved method constantly enriches and develops the theory and method of FMAGDM, and proposes a new idea for solving the FMAGDM problems. Finally, the results of a numerical example demonstrate the effectiveness of this new method. In our future research, we expect to develop further extensions of the improved method and apply it to other domains. Especially, we will focus on the more general methods for handling Multiple attribute decision making (MADM) problems.

\section{Acknowledgment}

This work is partially supported by the research fund of Sichun Key Laboratory of Intelligent
Network Information Processing (SGXZD100210), the National Natural Science Foundation (61175055, 61105059), Sichuan Key Technology Research and Development Program (2012GZ0019, 2011FZ0051) and the research fund of education department of Sichuan province (10ZC058) and the Fund of Science and technology innovation talent project of Sichuan Province (2011-039).

\section{References}

1. K. J. Arrow, "Social choice and individual values," New York, Wiley (1963).

2. S. M. Chen and S. J. Niou, "Fuzzy multiple attributes group decision making based on fuzzy induced OWA operators," Expert Systems with Applications, 38, 4097-4108 (2011).

3. F. Chiclana, E. Herrera-Viedma, F. Herrera and S. Alonso, "Some induced ordered weighted averaging operators and their use for solving group decisionmaking problems based on fuzzy preference relations," European Journal of Operational Research, 182, 383-399 (2007).

4. S. J. Chen and S. M. Chen, "A new method for handling multicriteria fuzzy decision-making problems using FN-IOWA operators," Cybernetics and Systems, 34, 109-137 (2003).

5. C. C. Chou, "Application of a Fuzzy MCDM Model to the Evaluation of Plant Location," International Journal of Innovative Computing, Information and Control, 6, 2581-2594 (2010).

6. X. W. Liao, Y. Li and B. Lu, "A model for selecting an ERP system based on linguistic information processing," Information Systems, 32, 1005-1017 (2007).

7. J. M. Merigo and M. Casanovas, "decision making with Uncertain Aggregation Operators Using the Dempster-Shafer Belief Structure," International Journal of Innovative Computing, Information and Control, 8, 1037-1061 (2012).

8. L. Martínez and F. Herrera, "An overview on the 2tuple linguistic model for Computing with Words in Decision Making: Extensions, applications and challenges," Information Sciences, 207, 1-18 (2012).

9. Z. X. Su, G. P. Xia, M. Y. Chen and L. Wang, "Induced generalized intuitionistic fuzzy OWA operator for multi-attribute group decision making," Expert Systems with Applications, 39, 1902-1910 (2012).

10. I. Palomares, J. Liu, Y. Xu and L. Martínez, "Modelling experts' attitudes in group decision making," Soft Computing, 16, 1755-1766 (2012).

11. Z. Pei and P. Shi, "Fuzzy risk analysis based on linguistic aggregation operators," International Journal of Innovative Computing, Information and Control, 7, 
7105-7118 (2011).

12. Z. Pei, "Fuzzy risk analysis based on linguistic information fusion," ICIC Express Letters, 3, 325-330 (2009).

13. R. M. Rodríguez, L. Martínez and F. Herrera, "Hesitant Fuzzy Linguistic Term Sets for Decision Making," IEEE Transactions on Fuzzy Systems, 20, 109119 (2012).

14. Y. J. Wang and H. S. Lee, "Generalizing TOPSIS for fuzzy multiple-criteria group decision making," Computers and Mathematics with Applications, 53, 17621772 (2007)

15. G. W. Wei, "A method for multiple attribute group decision making based on the ET-WG and ET-OWG operators with 2-tuple linguistic information," Expert Systems with Applications, 37, 7895-7900 (2010).
16. Z. S. Xu, "Induced uncertain linguistic OWA operators applied to group decision making," Information Fusion, 7, 231-238 (2006).

17. R. R. Yager, "On ordered weighted averaging aggregation operators in multicriteria decisionmaking," IEEE Transactions on Systems, Man, and Cybernetics, 18, 183-190 (1988).

18. R. R. Yager and D. P. Filev, "Induced ordered weighted averaging operators," IEEE Transactions on Systems, Man, and Cybernetics-Part B: Cybernetics, 29, 141-150 (1999).

19. Z. F. Zhang and X. N. Chu, "Fuzzy group decisionmaking for multi-format and multi-granularity linguistic judgments in quality function deployment," Expert Systems with Applications, 36, 9150-9158 (2009). 